\title{
GAS AND DUST IN THE BCD GALAXY VII ZW 403 (UGC 6456)
}

\author{
O.V. Egorov ${ }^{1, *}$ and T.A. Lozinskaya ${ }^{1, * *}$ \\ ${ }^{1}$ Sternberg Astronomical Institute, Universitetskiy pr. 13, Moscow, 119992 Russia
}

(Accepted by Astrophysical Bulletin)

\begin{abstract}
Based on the results of the earlier spectroscopic observations with the 6-m BTA telescope of the SAO RAS we refine the metallicity estimates of the complexes of ionized gas in the VII Zw 403 galaxy. Infrared observations from the Spitzer Space Telescope are used to search for a possible correlation of the mass fraction distribution of the polycyclic aromatic hydrocarbons (PAH) with the distribution of ionized and neutral hydrogen, and with the metallicity of gas in the HII regions of the galaxy.
\end{abstract}

\section{INTRODUCTION}

Detailed study of dwarf galaxies is important for understanding the origin and evolution of galaxies. According to current ideas, they play a role of building blocks, which are in their turn responsible for forming more massive systems $[1,2]$. The nearby blue compact dwarf galaxies (BCD) are of particular interest if the distances to them allow detailed studies of their structure, kinematics and emission spectra.

This paper continues a series of our investigations of ionized gas complexes, associated with the latest burst of star formation in the BCD galaxy VII Zw 403 (UGC 6456), presented by Arkhipova et al. [3] and Lozinskaya et al. [4].

VII $\mathrm{Zw} 403$ is one of the closest $\mathrm{BCD}$ galaxies $(D=4.5 \mathrm{Mpc})$, which has revealed several episodes of star formation of different intensity $[5,6]$. According to Lynds et al. [5], the most powerful burst of star formation occurred 600-800 Myr ago, the stars of this generation dominate in the galaxy. The age of the latest, fainter burst is about 4-10 Myr. Luz and Thuan [7] have classified VII Zw 403 as iE, the most numerous class of BCD galaxies, which is characterized by an irregular bright star-forming region near the center of an extended elliptical halo of old stars. Photometry of the stellar population of VII Zw 403 in the near IR confirmed that the region of recent star formation is surrounded by an extended "old" stellar halo [8]. The latest burst of star formation encompassed the central region of the galaxy, spanning about $1 \mathrm{kpc}$ in the direction of a giant cloud of neutral hydrogen with the highest radiation density, surrounded by an extended HI halo sized $3.6^{\prime} \times 2.9^{\prime}$ $(4.7 \times 3.8 \mathrm{kpc})[9,10]$. Several sites of this latest star formation episode are observed: the youngest massive stars form compact OB associations № 1-6 (for consistency, we use the nominations of associations and HII regions according to [5]).

* Electronic address: morzedon@gmail.com

** Electronic address: lozinsk36@mail.ru
The ionized gas is concentrated in the same central region; the associations are linked with bright HII regions sized $80-150 \mathrm{pc}$ surrounded by the faint diffuse $\mathrm{H}_{\alpha}$ emission $[5,11,12]$. The HST observations have disclosed a shell-like structure of several bright HII regions; Lynds et al. [5] measured the shell's expansion velocity of $50-70 \mathrm{~km} / \mathrm{s}$, however, Lozinskaya et al. [4] did not confirm these results. Silich et al. [13] found the traces of a faint giant ring sized $D \simeq 500 \mathrm{pc}$ in the $\mathrm{H}_{\alpha}$ emission of diffuse gas.

In our paper [4] we have investigated the structure and kinematics of ionized gas in VII $\mathrm{Zw}_{\mathrm{w}} 403$. In addition to the previously known bright HII regions and traces of a faint giant ring, many new faint diffuse and arc-shaped structures were found, and a "fine structure" of the giant ring was identified. We found no evidence of the shell-like regions expanding with the velocity of $50-70 \mathrm{~km} / \mathrm{s}$, which was reported in [5], but instead we detected a clear line broadening (FWHM up to $60-120 \mathrm{~km} / \mathrm{s}$ ) in the region of weak diffuse emission outside the bright HII regions. In the brightest shell № 1 around the richest and youngest association № 1 faint details are revealed in the wings of the [OIII] line at the velocities of up to $-200 \div-300 \mathrm{~km} / \mathrm{s}$, in the wings of $\mathrm{H}_{\alpha}$ line - at the velocities of up to $-350 \mathrm{~km} / \mathrm{s}$, and up to $550-600 \mathrm{~km} / \mathrm{s}$ from the line center [4]. Such velocities in VII Zw 403 were discovered for the first time and are a clear evidence of gas acceleration at the shock front. The kinematic age of the bright shells, corresponding to our estimates of their average expansion velocities $(20 \mathrm{~km} / \mathrm{s}$ or below) is at least $2-4$ Myr, which agrees well with the age of their central OB associations, reported in [5]. Faint extended filamentary and diffuse regions of ionized gas that can be observed in the entire central part of the galaxy, as well as the giant HII ring can be related to the older stellar population of the latest star formation burst (with the age of $10 \mathrm{Myr}$ according to [5]).

In [3], based on the observations of VII Zw 403 at the 6-m BTA telescope of the SAO RAS with the panoramic Multipupil Fiber Spectrograph (MPFS) and with the SCORPIO focal reducer in the slit spectrograph mode, we have analyzed the gas emis- 
sion spectrum and determined the relative oxygen abundance in the regions № $1,3,4$, and the nitrogen and sulfur abundances in all regions. To our knowledge, these estimates of metallicity in individual regions of ionized gas in VII Zw 403 were the first, all the previously published values are averaged across the galaxy.

The startup of the Spitzer Space Telescope has opened new avenues for studying the dust component of the interstellar medium of galaxies. The polycyclic aromatic hydrocarbons (PAH): the macromolecules consisting of tens of and hundreds of atoms, mostly hydrogen and carbon [14], are of particular interest. The mechanism of formation and destruction of PAH molecules is not yet completely understood, the discussed possibilities are: their formation in the atmospheres of carbon-rich AGB and post-AGB stars or in the dense molecular clouds, and the destruction of these molecules by the shock waves or UV emission of hot stars (see, e.g., Sandstrom et al. [15] and references therein).

To clarify the mechanisms of formation and destruction of PAHs and their relationship with the physical parameters and metallicity of gas in the galaxies, Sandstrom et al. [15], based on the Spitzer observations have investigated the dust component of the nearby dIrr galaxy Small Magellanic Cloud. Our own detailed analysis of the infrared emission of dIrr galaxy IC 10 has shown that the results of observations are better consistent with the assumption that the PAHs form in the molecular clouds and are destroyed by the UV emission, rather than being formed in the atmospheres of carbon-rich stars [16]. To elucidate the nature of PAHs, it may be important to refer to the observed deficiency of radiation of these molecules in the galaxies with low metallicity. According to Drain et al.[17], this deficiency is related to the shortage of PAHs themselves, rather than to the less efficient excitation of the IR transitions in them. In our work [16], based on the observations of HII regions in the IC 10 galaxy, we for the first time suspected a correlation between the mass fraction of dust contained in the PAHs $\left(q_{\mathrm{PAH}}\right)$, and metallicity of gas at the level of individual HII regions. However, the accuracy of the discovered dependence was found to be insufficient to consider this correlation reliable, and we find it interesting to test it on the observations of other galaxies.

In this paper we refine the abundances of oxygen, nitrogen and sulfur in certain HII regions from the results of previous observations of VII Zw 403 with the 6-m BTA telescope of the Special Astrophysical Observatory of Russian Academy of Sciences (SAO RAS) [3, 4]. Based on the archive data of the Spitzer Space Telescope we investigate the infrared emission of the galaxy with the aim to analyze the distribution of the PAHs and find a possible correlation of $q_{\mathrm{PAH}}$ with the radiation brightness of the ionized and neutral gas, as well as with the metallicity of HII regions.

The following sections describe the observations we used and present the obtained results, and the conclusion summarizes our main findings.

The distance to VII $\mathrm{Zw} 403$ is adopted as $D=4.5 \mathrm{Mpc}[5,6]$, which corresponds to an angular scale of $22 \mathrm{pc} /{ }^{\prime \prime}$.

\section{OBSERVATIONS USED}

\subsection{Spectral Observations}

Spectral observations of VII Zw 403 were performed at the 6-meter SAO RAS BTA telescope with the SCORPIO focal reducer ${ }^{1}[18]$ in the spectrograph slit mode, as well as with the panoramic Multipupil Fiber Spectrograph (MPFS). Both series of observations, carried out in 2003 and 2004 are described in detail in our paper [3], we shall only briefly summarize the basic data here.

In the observations with a slit spectrograph, the slit length was about $6^{\prime}$, its width was $1^{\prime \prime}$, the scale along the slit $-0.36^{\prime \prime} /$ pixel. Two spectrograms were obtained, the spectrograph slit localization is shown in Fig. 1. Slit 1 passed through the complexes № 24, Slit 2-through the complexes № 1 and 5. For two slit positions we obtained the spectra in the ranges of 6270-7300 A and 4800-5600 A, the seeing was about $2^{\prime \prime}$, the exposure ranged from 800 to $1800 \mathrm{~s}$. The data reduction was done using the standard method, the spectrophotometric standard AGK $+81^{\circ} 266$ was applied to bind to the energy scale. The emission line intensities were determined using a singlecomponent Gaussian approximation of profiles.

In the observations with the MPFS ${ }^{2}$ [19] the spectra from 256 spatial elements were simultaneously registered, making up the matrix in the picture plane sized $16 \times 16$ elements, where the angular size of the pixel is $1^{\prime \prime}$. The spectra with the resolution of about $8 \AA$ were obtained in the range of 4250-7200 $\AA$ for seven fields in the central region of the galaxy, taken with a mutual offset. The total time of exposures for each field varied within $600-1800 \mathrm{~s}$ with the average seeing of $1.7^{\prime \prime}-2^{\prime \prime}$.

The observations were reduced using the software packages developed in the SAO RAS Laboratory of the Spectroscopy and Photometry of Extragalactic Objects, running under IDL. The spectra

\footnotetext{
1 http://www.sao.ru/hq/lsfvo/devices/scorpio/ /scorpio.html

2 http://www.sao.ru/hq/lsfvo/devices/mpfs/ /mpfs_main.html
} 


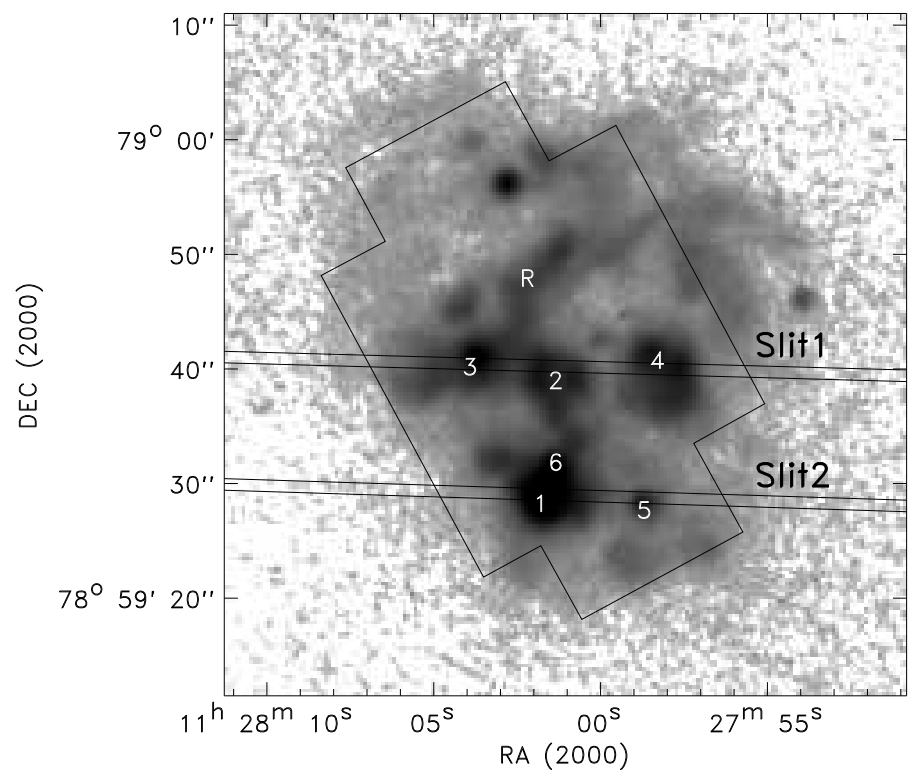

Figure 1. The region of the latest star formation burst in VII $\mathrm{Zw}_{\mathrm{w}} 403$ viewed in the $\mathrm{H}_{\alpha}$ line. The localization of the spectrograph slit and a part of the galaxy, overlapped with the MPFS observations are shown. The numbers indicate the HII complexes (according to the markings by Lynds et al. [5]), the letter $\mathrm{R}$ marks the brightest part of the giant ring.

GRW $+70^{\circ} 5824$ were used as the spectrophotometric standard. The result of data reduction is the data cube, in which each pixel of the image sized $16^{\prime \prime} \times 16^{\prime \prime}$ corresponds to the spectrum of 2048 elements. After the initial reduction the data cubes for all seven fields were aligned and stacked, so that the size of the resulting mosaic amounted to $49^{\prime \prime} \times 31^{\prime \prime}$; the corresponding region of the galaxy is shown in Fig. 1.

\subsection{Archival Observational Data of the Spitzer Space Telescope}

We used the archival observational data of the galaxy VII $\mathrm{Zw} 403$ with the IRAC and MIPS instruments obtained within the program called the Starburst Activity in Nearby Galaxies ${ }^{3}$. The data were downloaded from the archive using the Spitzer Heritage Archive system ${ }^{4}$. The images of the galaxy were obtained in seven wavelengths: $3.6 \mu \mathrm{m}, 4.5 \mu \mathrm{m}$, $5.8 \mu \mathrm{m}, 8.0 \mu \mathrm{m}$ from the observations with the IRAC instrument, and $24 \mu \mathrm{m}, 70 \mu \mathrm{m}, 160 \mu \mathrm{m}-$ with the MIPS instrument. The images were combined in mosaic using the MOPEX software ${ }^{5}$; the images in the $70 \mu \mathrm{m}$ and $160 \mu \mathrm{m}$ bands were processed using the

\footnotetext{
3 Spitzer Proposal, ID 59 (2003)

${ }^{4}$ http://sha.ipac.caltech.edu

5 http://ssc.spitzer.caltech.edu/dataanalysistools/ /tools/mopex/
}

GeRT software package ${ }^{6}$. For the analysis of mosaic images we used our own procedures written in IDL. In addition, in order to determine the integral fluxes as the level of background emission in all infrared bands, we used the mean intensity in the positions, distant from the main star-forming regions of the galaxy. In our case, this method is applicable owing to the small angular size of VII Zw 403; the galaxy occupied a small part of the field in the images.

\section{RESULTS}

Figure 1 demonstrates the image of the starforming complex of VII $\mathrm{Zw} 403$ in the $\mathrm{H}_{\alpha}$ line, obtained from our observations with the 6-m telescope [4]. The figure shows the localization of two slits and the mosaic of fields, observed with the MPFS; the numbers denote the numbers of HII regions based on the markings by Lynds et al.[5], the letter $\mathrm{R}$ marks the above-mentioned gigantic ring.

Determining the fluxes in the emission lines, we averaged individual regions to increase the signalto-noise ratio.

To refine the metallicity in HII regions of the galaxy we used the line intensities, previously measured in [3], as well as the data adopted from Lynds et al. [5]. In this paper we additionally

\footnotetext{
6 http://ssc.spitzer.caltech.edu/dataanalysistools/ /tools/gert/
} 
take into account the relative intensities of weaker lines: [SIII] $\lambda 6312 \AA / \mathrm{H}_{\alpha}$ for the regions № $1-5$ from the observations with a slit spectrograph, and [OIII $] \lambda 4363 \AA / \mathrm{H}_{\beta}$ for the region № 1 from the MPFS observations. All the relative line intensities used (not corrected for the interstellar absorption) are listed in Table 1.

\subsection{Metallicity of Ionized Gas in the Galaxy}

In [3] we estimated the relative abundances of oxygen, nitrogen and sulfur based on the assumption that inside the HII regions these elements predominantly exist in the OIII, NII, SII states, respectively. The rationale for this followed from the analysis of radial distribution of relative intensities of the corresponding lines inside the brightest region № 1 . In this paper, we estimate the metallicity of gas from the line intensities listed in Table 1, taking into account the other ionization stages as well. We applied the well-known methods for estimating the oxygen, nitrogen and sulfur abundances: the classic "direct" $T_{e}$-method (Izotov et al. [20]), and a number of empirical methods. The results are presented in Table 2 with the indications of the methods used.

\subsubsection{The $T_{e}$-Method}

In order to use the $T_{e}$-method (Izotov et al. [20]) we have to know the temperature $T_{e}$ and density $n_{e}$ of gas (but the latter has little effect on the result). The density is found from the lines of [SII] $\lambda 6717 / \lambda 6731$; the temperature was estimated with the Five Level program [21] from the line intensity ratio of [OIII] $(\lambda 4959 \AA+\lambda 5007 \AA) / \lambda 4363 \AA$ Alines. Our observations allowed to confidently measure the intensity of the [OIII] $\lambda 4363 \AA$ line only for region № 1 ; the $T_{e}$ value we got coincides with the estimate by Lynds et al. [5].

To determine the temperature in the HII regions № 3 and 4, we used the observations from [5]; while for the regions № 2 and 5, where the line [OIII] $\lambda 4363 \AA$ was never measured, we adopted the temperature $T_{e}=14500$, measured for region № 1 .

Knowing $T_{e}$ and $n_{e}$, we determined the abundance of ions $\mathrm{O}^{+}, \mathrm{O}^{2+}$ using the relations (3) and (5) from [20]. The full oxygen abundance is found in the assumption that the oxygen in the HII regions predominantly exists in the OII and OIII states. The abundances of the nitrogen and sulfur ions are found from the relations (6), (8) and (9) from [20]. To get the full abundance of these elements, we calculated the ionization correction factors $(I C F)$ for the unobserved ionization stages from the relations (18) and (20) for the mean metallicity of $12+\log (\mathrm{O} / \mathrm{H})=7.6$. To determine the abundances of $\mathrm{O}^{+}, \mathrm{N}^{+}, \mathrm{S}^{+}$we adopted $T_{e}$ in the emission region of these ions, computed from relation (14), and to determine the $\mathrm{S}^{2+}$ abundance, the $T_{e}$ was computed using relation (15) from [20].

The estimates of the relative abundances of oxygen, nitrogen and sulfur by the $T_{e}$-method are presented in Table 2 and indicated by the index " $T_{e}$ "; the metallicity measurement errors listed do not take into account the temperature estimation error.

\subsubsection{Empirical methods}

A series of methods is currently widely used to estimate the abundances of chemical elements in the HII regions. These methods apply the empirical relationships between the metallicity and relative line intensities in the spectrum. Their accuracy is generally inferior to that of the "direct" $T_{e}$-method.

However, since we determined $T_{e}$ from our observations only for the region № 1 , we used the data from [5] for the regions № 3 and 4, and for regions № 2 and 5 there are no estimates of $T_{e}$ at all, we hence found it useful to apply the empirical methods for estimating their metallicity.

We used the long-known empirical P-method (Pilyugin, Thuan [22]), based on the dependence of oxygen abundance on the excitation parameter $P$ and the parameter $R_{23}$, which are determined from the relative intensities of the [OII] and [OIII] lines. As a result, we found that the estimates computed by this technique and using the $T_{e}$-method for different HII regions are in good agreement.

An empirical dependence proposed by Pettini and Pagel [23] has an advantage, which is based on the relationship of bright and closely spaced lines $\left([\mathrm{OIII}] / \mathrm{H}_{\beta}\right.$ and $\left.[\mathrm{NII}] / \mathrm{H}_{\alpha}\right)$, i.e. it is insensitive to absorption. However, the values of the O3N2 parameter, used in this dependence, turned out to be outside the range of confident method applicability for three regions of VII Zw 403, and at its boundary for two other regions.

We as well used nine empirical relations for various line intensity ratios, proposed in [24], but they gave a large (approximately $\pm 0.5 \mathrm{dex}$ ) scatter of estimates for the same region in different lines. At that, the mean metallicity estimates based on different dependences are consistent with the estimates of the $T_{e}$-method.

Pilyugin et al. [25], and Pilyugin and Mattson [26] have proposed the most accurate empirical methods for estimating the metallicity. The accuracy of estimates, obtained applying two techniques from the former work, is, according to the authors, $0.075 \mathrm{dex}$ for the oxygen abundance and 0.05 dex for the nitrogen abundance; the accuracy of methods proposed in the latter work is evaluated as 0.077 dex 
Table 1. Relative line intensities in the spectra of five bright HII regions

\begin{tabular}{|c|c|c|c|c|c|c|}
\hline Lines & № 1 & № 2 & № 3 & № 4 & № 5 & reference \\
\hline [OII] $3729 / 3727$ & $1.39 \pm 0.11$ & $1.43 \pm 0.14$ & $1.50 \pm 0.08$ & $1.37 \pm 0.10$ & $1.80 \pm 0.23$ & [5] \\
\hline$[\mathrm{OII}](3727+29) / \mathrm{H}_{\beta}$ & $0.59 \pm 0.02$ & $2.26 \pm 0.11$ & $1.18 \pm 0.03$ & $1.71 \pm 0.06$ & $2.20 \pm 0.12$ & [5] \\
\hline$[\mathrm{OIII}](5007) / \mathrm{H}_{\beta}$ & $3.89 \pm 0.01$ & $1.65 \pm 0.03$ & $2.68 \pm 0.01$ & $2.59 \pm 0.03$ & $1.28 \pm 0.03$ & [5] \\
\hline [OIII $](5007) / \mathrm{H}_{\beta}$ & $3.43 \pm 0.05$ & $1.72 \pm 0.02$ & $2.85 \pm 0.03$ & $2.78 \pm 0.03$ & $1.69 \pm 0.01$ & $*$ \\
\hline [OIII $](5007) / \mathrm{H}_{\beta}$ & $3.86 \pm 0.02$ & $1.79 \pm 0.04$ & $2.85 \pm 0.02$ & $2.82 \pm 0.04$ & $1.63 \pm 0.07$ & ** \\
\hline [OIII $](4363) / \mathrm{H}_{\beta}$ & $0.064 \pm 0.002$ & - & $0.049 \pm 0.006$ & $0.063 \pm 0.009$ & - & [5] \\
\hline [OIII] $(4363) / \mathrm{H}_{\beta}$ & $0.067 \pm 0.009$ & - & - & - & - & $* *$ \\
\hline$[\mathrm{OI}](6300) / \mathrm{H}_{\beta}$ & $0.011 \pm 0.003$ & $0.04 \pm 0.01$ & $0.044 \pm 0.005$ & - & - & 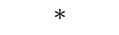 \\
\hline [SIII] $(6312) / \mathrm{H}_{\alpha}$ & $0.0066 \pm 0.0014$ & $0.0073 \pm 0.0038$ & $0.0133 \pm 0.0052$ & $0.0087 \pm 0.0031$ & $0.0072 \pm 0.0059$ & * \\
\hline$[\mathrm{SII}](6717+31) / \mathrm{H}_{\alpha}$ & $0.066 \pm 0.001$ & $0.137 \pm 0.001$ & $0.123 \pm 0.001$ & $0.096 \pm 0.001$ & $0.137 \pm 0.003$ & * \\
\hline$[\mathrm{SII}](6717+31) / \mathrm{H}_{\alpha}$ & $0.051 \pm 0.001$ & $0.140 \pm 0.008$ & $0.110 \pm 0.003$ & $0.092 \pm 0.005$ & $0.137 \pm 0.008$ & ** \\
\hline$[\mathrm{SII}](6717) / \mathrm{H}_{\alpha}$ & $0.028 \pm 0.001$ & $0.07 \pm 0.012$ & $0.06 \pm 0.003$ & $0.056 \pm 0.005$ & $0.076 \pm 0.09$ & ** \\
\hline$[\mathrm{NII}](6548+84) / \mathrm{H}_{\alpha}$ & $0.024 \pm 0.001$ & $0.032 \pm 0.001$ & $0.024 \pm 0.001$ & $0.025 \pm 0.003$ & $0.038 \pm 0.002$ & * \\
\hline [NII] $(6584) / \mathrm{H}_{\alpha}$ & $0.013 \pm 0.001$ & $0.027 \pm 0.001$ & $0.022 \pm 0.001$ & $0.021 \pm 0.001$ & $0.034 \pm 0.002$ & $*$ \\
\hline $\mathrm{H}_{\gamma} / \mathrm{H}_{\beta}$ & $0.44 \pm 0.01$ & $0.50 \pm 0.01$ & $0.42 \pm 0.01$ & $0.47 \pm 0.01$ & $0.51 \pm 0.02$ & [5] \\
\hline $\mathrm{H}_{\beta} / \mathrm{H}_{\alpha}$ & $0.313 \pm 0.002$ & $0.304 \pm 0.005$ & $0.307 \pm 0.002$ & $0.309 \pm 0.004$ & $0.255 \pm 0.01$ & ** \\
\hline
\end{tabular}

and 0.110 dex for oxygen and nitrogen, respectively.

The results obtained for the HII regions of VII Zw 403 based on the empirical dependences from these papers are presented in Table 2 .

The technique from [25] allows us to estimate the relative oxygen and nitrogen abundances from the ratio of intensities of the following bright lines:

$([\mathrm{OIII}] \lambda 4959+5007 \AA) / \mathrm{H}_{\beta}$,

([OII] $\lambda 3727+3729 \AA) / \mathrm{H}_{\beta}$,

([NII] $\lambda 6548+6584 \AA) / \mathrm{H}_{\beta}$.

The authors designate this method as ON-method. When applying the ONS-method, another ratio ([SII] $\lambda 6717+6731 \AA) / \mathrm{H}_{\beta}$ is as well in use. We have applied both methods; to estimate the relative abundances of oxygen and nitrogen via the ONS-method we used the ratios (17) and (18) from [25]; via the ON-method-the ratios (19) and (20) were applied. The results obtained are listed in Table 2, indicated by the indices "ONS" and "ON", respectively.

The NS-method presented in [26] possesses a certain advantage for us as it does not require the measurement of intensities of ([OII] $\lambda 3727+3729 \AA$ ) lines. Applying this method, from the relative intensities of the following bright lines:

$([\mathrm{OIII}] \lambda 4959+5007 \AA) / \mathrm{H}_{\beta}$,

([NII] $\lambda 6548+6584 \AA) / \mathrm{H}_{\beta}$, and

([SII] $\lambda 6717+6731 \AA) / \mathrm{H}_{\beta}$, we have found the relative oxygen abundance from relation (8), and relative nitrogen abundance from relation (9) in [26]. The results are listed in Table 2 and indicated by the "NS" indices.

We further use the metallicity estimates of individual HII regions, listed in Table 2, obtained by the direct $T_{e}$-method and by the most accurate empirical methods [25, 26], which are yielding results in good agreement with the $T_{e}$-method.

In particular, for regions № 1, 3, 4 we adopted the estimates of the $T_{e}$-method, while for regions № 2,5 , in which there are no $T_{e}$ measurements, we averaged the listed values that were determined with different techniques.

As we can see from Table 2 , the metallicity in individual HII regions does not reveal strong variations and is well consistent with the mean values over the galaxy, obtained by other authors. According to our estimate, the average metallicity in VII Zw 403 amounts to $12+\log (\mathrm{O} / \mathrm{H})=7.66 \pm 0.03$; according to Izotov and al. [27] it is equal to $12+$ $\log (\mathrm{O} / \mathrm{H})=7.73 \pm 0.01$; Nagao et al. [24] used the line intensities measured in [27] to obtain $12+\log (\mathrm{O} / \mathrm{H})=7.7 \pm 0.01$; while Schulte-Ladbeck et al. [8] give $12+\log (\mathrm{O} / \mathrm{H})=7.63-7.71$.

The abundance of nitrogen in different regions is also consistent with the average across the galaxy: $12+\log (\mathrm{N} / \mathrm{H})=6.19 \pm 0.08$ according to [27].

Our estimates of the sulfur abundance are higher than the average across the galaxy: $12+\log (\mathrm{S} / \mathrm{H})=6.16 \pm 0.04$ according to [27], but given the measurement errors they are consistent with this value for all regions, except region ㄲo 3 .

The nitrogen abundance, averaged across the galaxy, according to our evaluation is $12+\log (\mathrm{N} / \mathrm{H})=6.22 \pm 0.19$, the sulfur abundance amounts to $12+\log (\mathrm{S} / \mathrm{H})=6.27 \pm 0.14$. Note that for VII Zw 403 we found practically equal abundances of nitrogen and sulfur in the HII regions. Similar abundances of these elements are observed in other galaxies, such as the Magellanic Clouds [28] and the NGC 3109 [29]. The galaxy NGC 3109 is the closest analogue of VII Zw 403 based on the abundance of $\mathrm{O}, \mathrm{N}$ and $\mathrm{S}$.

\subsection{The Dust and Polycyclic Aromatic Hydrocarbons in VII $Z_{W} 403$}

Our particular interest to the IR observations of VII Zw 403 stems from the fact that our analysis of 
Table 2. Metallicity of HII regions

\begin{tabular}{l|c|c|c|c|c}
\hline \multirow{2}{*}{ Estimate } & \multicolumn{5}{|c}{ HII regions } \\
\cline { 2 - 6 } & №1 & №2 & №3 & №4 & №5 \\
\hline$N_{e}(\mathrm{SII}) \mathrm{cm}^{-3}$ & 57 & 66 & 57 & 110 & - \\
$T_{e}(\mathrm{OIII}) \mathrm{K}$ & 14500 & - & 14900 & 16200 & - \\
\hline $12+\log (\mathrm{O} / \mathrm{H})\left(T_{e}\right)$ & $7.69 \pm 0.01$ & $7.69 \pm 0.03$ & $7.66 \pm 0.01$ & $7.64 \pm 0.02$ & $7.68 \pm 0.03$ \\
$12+\log (\mathrm{O} / \mathrm{H})(\mathrm{ONS})$ & $7.72 \pm 0.02$ & $7.75 \pm 0.03$ & $7.68 \pm 0.02$ & $7.71 \pm 0.03$ & $7.79 \pm 0.04$ \\
$12+\log (\mathrm{O} / \mathrm{H})(\mathrm{ON})$ & $7.80 \pm 0.02$ & $7.55 \pm 0.02$ & $7.61 \pm 0.02$ & $7.65 \pm 0.04$ & $7.59 \pm 0.03$ \\
$12+\log (\mathrm{O} / \mathrm{H})(\mathrm{NS})$ & $7.77 \pm 0.01$ & $7.56 \pm 0.01$ & $7.62 \pm 0.01$ & $7.67 \pm 0.03$ & $7.60 \pm 0.02$ \\
\hline $12+\log \left(\mathrm{N}^{+} / \mathrm{H}\right)$ & $5.72 \pm 0.02$ & $5.86 \pm 0.02$ & $5.72 \pm 0.02$ & $5.70 \pm 0.05$ & $6.01 \pm 0.03$ \\
$I C F\left(\mathrm{~N}^{+}\right)$ & $6.82 \pm 0.22$ & $1.75 \pm 0.10$ & $3.27 \pm 0.08$ & $2.45 \pm 0.09$ & $1.70 \pm 0.11$ \\
$12+\log (\mathrm{N} / \mathrm{H})\left(T_{e}\right)$ & $6.55 \pm 0.02$ & $6.10 \pm 0.03$ & $6.23 \pm 0.02$ & $6.09 \pm 0.05$ & $6.24 \pm 0.04$ \\
$12+\log (\mathrm{N} / \mathrm{H})(\mathrm{ONS})$ & $6.48 \pm 0.03$ & $6.13 \pm 0.04$ & $6.09 \pm 0.03$ & $6.11 \pm 0.07$ & $6.22 \pm 0.06$ \\
$12+\log (\mathrm{N} / \mathrm{H})(\mathrm{ON})$ & $6.56 \pm 0.02$ & $6.01 \pm 0.03$ & $6.09 \pm 0.02$ & $6.09 \pm 0.06$ & $6.09 \pm 0.04$ \\
$12+\log (\mathrm{N} / \mathrm{H})(\mathrm{NS})$ & $6.32 \pm 0.02$ & $6.07 \pm 0.02$ & $6.09 \pm 0.02$ & $6.18 \pm 0.04$ & $6.13 \pm 0.03$ \\
$12+\log \left(\mathrm{S}^{+} / \mathrm{H}\right)$ & $5.39 \pm 0.01$ & $5.73 \pm 0.03$ & $5.60 \pm 0.01$ & $5.50 \pm 0.02$ & $5.79 \pm 0.03$ \\
$12+\log \left(\mathrm{S}^{2+} / \mathrm{H}\right)$ & $6.07 \pm 0.09$ & $6.12 \pm 0.23$ & $6.23 \pm 0.16$ & $5.96 \pm 0.15$ & $6.12 \pm 0.35$ \\
$I C F\left(\mathrm{~S}^{+}+\mathrm{S}^{2+}\right)$ & $1.32 \pm 0.02$ & $1.05 \pm 0.01$ & $1.10 \pm 0.01$ & $1.06 \pm 0.01$ & $1.05 \pm 0.01$ \\
$12+\log (\mathrm{S} / \mathrm{H})$ & $6.27 \pm 0.07$ & $6.29 \pm 0.16$ & $6.36 \pm 0.13$ & $6.11 \pm 0.11$ & $6.31 \pm 0.24$ \\
\hline
\end{tabular}

the dust component of the Irr galaxy IC 10 resulted in shedding some light on the as yet unclear mechanisms of the formation and destruction of the PAH molecules [16]. We will hence attempt to similarly consider the IR observations of VII Zw 403.

Figure 2 demonstrates the maps of the infrared emission distribution in VII Zw 403 in the $8 \mu \mathrm{m}$ and $24 \mu \mathrm{m}$ bands, constructed from the Spitzer observational data, combined with the $\mathrm{H}_{\alpha}$ image.

The photometry of VII Zw 403 in different IR bands from the Spitzer observations was earlier conducted by Engelbracht et al. [30]. We have performed the subsequent aperture photometry of the entire galaxy based on the same data, the results of which are listed Table 3. Our estimates somewhat vary from the results in [30], the differences are most probably due to different values of the adopted background level in the images; however, these differences have practically no influence on the results discussed below.

In the analysis of the infrared radiation Draine and $\mathrm{Li} \mathrm{[31]} \mathrm{proposed} \mathrm{to} \mathrm{parameterize} \mathrm{the} \mathrm{UV} \mathrm{field}$ of the galaxy as the sum of the "minimal" diffuse UV field $U_{\mathrm{min}}$, filling the largest part of its volume, and the more intensive UV field with a power law energy distribution, affecting only the mass fraction $\gamma$ of all the dust in the galaxy. $U_{\min }$, expressed in the units of average UV-field of our Galaxy, characterizes the overall level of star formation in the studied system, and $\gamma$ allows to estimate what fraction of the galaxy's matter is involved in the current processes of star formation. Draine and Li [31] give the algorithm for estimating the parameters of the galaxy from the observations in the infrared bands of $8 \mu \mathrm{m}, 24 \mu \mathrm{m}, 70 \mu \mathrm{m}$ and $160 \mu \mathrm{m}$ (the data for the $3.6 \mu \mathrm{m}$ band is used to remove the contribution of the stellar radiation). We used the results of photometry of VII $\mathrm{Zw} 403$ to apply the meth- ods proposed in [31] and try to determine $U_{\min }, \gamma$, and $q_{\mathrm{PAH}}$, or the mass fraction of dust contained in the PAHs. Unfortunately, the values of the parameters found for VII $\mathrm{Zw}$ 403, that appear in the models from [31], are beyond the limits, for which these models are calculated. Therefore, we could not determine the $U_{\min }$ and $\gamma$, we only got the upper limit of $q_{\mathrm{PAH}}<0.5 \%$ and the lower limit of $U_{\min }>25$. This value of $U_{\min }$ indicates a high level of star formation in VII Zw 403, which is natural for $\mathrm{BCD}$ galaxies. A low abundance of PAHs is consistent with the hypothesis of the destruction of these molecules under the effect of UV radiation in the regions of star formation near the hot stars.

A number of authors, in particular Sandstrom et al. [15] note the possibility of using the flux ratio at the wavelengths of $8 \mu \mathrm{m}$ and $24 \mu \mathrm{m}$ as a local indicator of the mass fraction of $q_{\mathrm{PAH}}$. Based on the correlation that the above authors have found between the $q_{\mathrm{PAH}}$ and the ratio $F_{8 \mu \mathrm{m}} / F_{24 \mu \mathrm{m}}$, we have analyzed the distribution of $q_{\mathrm{PAH}}$ in VII Zw 403 and tried to compare it with the distribution of ionized and neutral gas in the galaxy.

Figure 3 demonstrates the maps of distribution of the flux ratio $F_{8 \mu \mathrm{m}} / F_{24 \mu \mathrm{m}}$ with the superimposed isophotes in the lines of $\mathrm{H}_{\alpha}$ (left) and $\mathrm{HI} 21 \mathrm{~cm}$ (right). We used the isophotes in the $21 \mathrm{~cm}$ line from the VLA observations, presented by Ashley and Simpson [10].

It follows from Fig. 3 that large variations of the flux ratio $F_{8 \mu \mathrm{m}} / F_{24 \mu \mathrm{m}}$ are not revealed in the central part of VII Zw 403, except for the bright western region. However, we clearly see that this ratio is increased at the borders and outside the HII regions, and declines in their central parts. This may be due to the destruction of the PAH molecules in the bright regions of ionized gas under the effect of ultraviolet radiation of the central OB associations. 

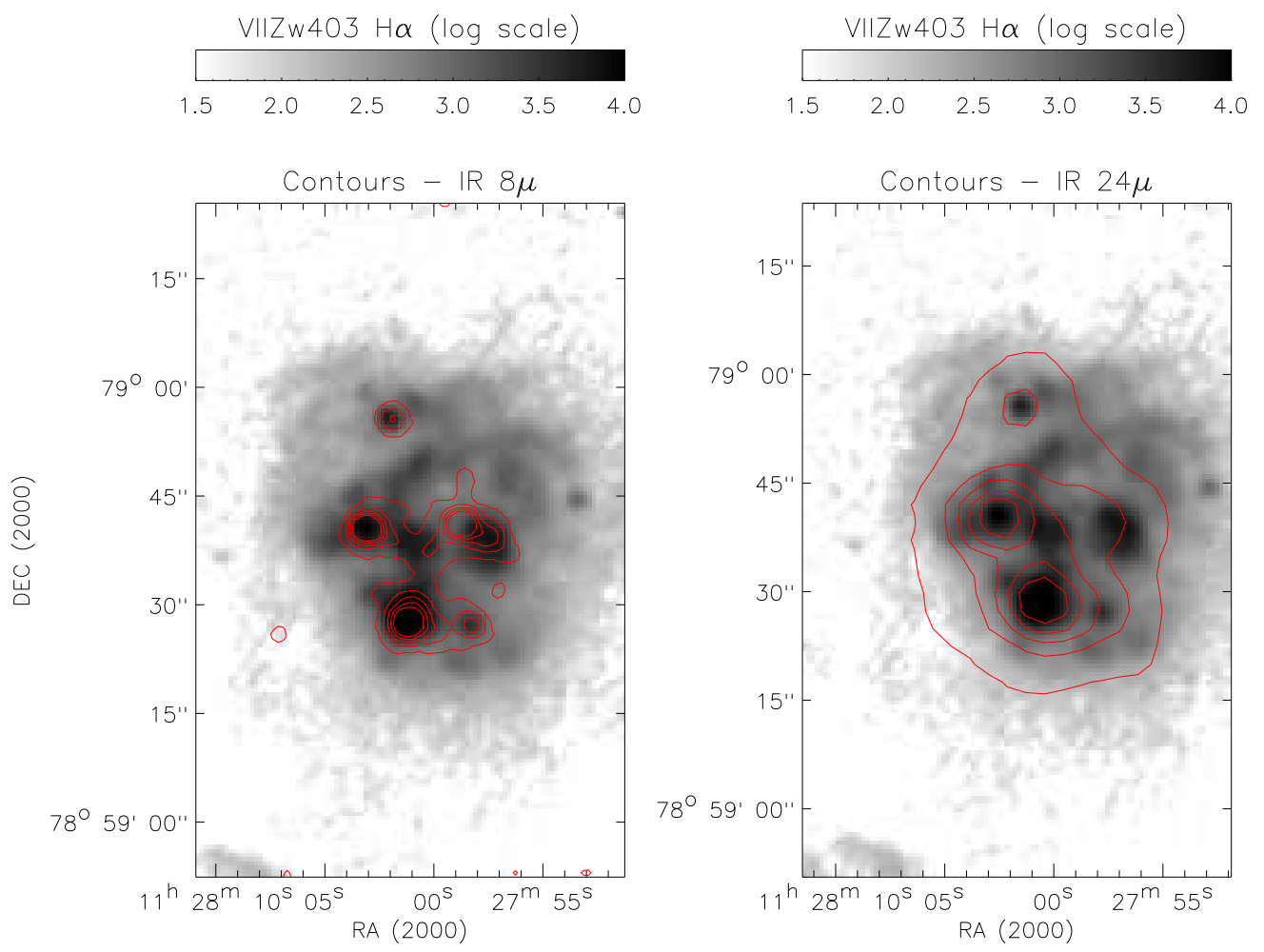

Figure 2. An image of the star-forming region of VII $\mathrm{Zw} 403$ in the $\mathrm{H}_{\alpha}$ line with the superimposed contours in different IR range bands: left $-8 \mu \mathrm{m}$, right $-24 \mu \mathrm{m}$. The isophotes, corresponding to the levels of $0.1,0.2,0.3,0.4$ and $0.5 \mathrm{MJy} / \mathrm{sr}$ for $8 \mu \mathrm{m}$ and $0.2,0.9,1.6,2.3,3.0 \mathrm{MJy} / \mathrm{sr}$ for $24 \mu \mathrm{m}$ are shown.

Table 3. The results of photometry of the images from the Spitzer Space Telescope

\begin{tabular}{c|c|c|c|c|c|c}
\hline$F_{3.6 \mu \mathrm{m}}, \mathrm{mJy}$ & $F_{4.5 \mu \mathrm{m}}, \mathrm{mJy}$ & $F_{5.8 \mu \mathrm{m}}, \mathrm{mJy}$ & $F_{8.0 \mu \mathrm{m}}, \mathrm{mJy}$ & $F_{24 \mu \mathrm{m}}, \mathrm{mJy}$ & $F_{70 \mu \mathrm{m}}, \mathrm{Jy}$ & $F_{160 \mu \mathrm{m}}, \mathrm{Jy}$ \\
\hline $3.27 \pm 0.02$ & $1.94 \pm 0.02$ & $2.02 \pm 0.06$ & $3.58 \pm 0.07$ & $28.4 \pm 0.7$ & $0.355 \pm 0.003$ & $0.123 \pm 0.003$ \\
\hline
\end{tabular}

Due to the low spatial resolution we could not discern in VII Zw 403 a distinct correlation of the PAH distribution with the envelopes of neutral hydrogen, similar to that observed in IC 10 [16]. As follows from Fig. 3 , the highest value of $F_{8 \mu \mathrm{m}} / F_{24 \mu \mathrm{m}}$, corresponding to the largest mass fraction of $\mathrm{PAH}$, occurs in the direction of the western part of the dense HI cloud. It is possible that in the direction of the southern part of the cloud, the PAH molecules are destroyed by the ultraviolet emission of the OB associations located here. Violent ultraviolet emission is as well revealed by the brightest in galaxy shell region № 1 located here, see Fig. 1.

The galaxies of low metallicity are revealing a deficiency of PAH radiation, linked with a shortage of these macromolecules (see Drain et al.[17] and references therein). In the galaxies with the oxygen abundance of $12+\log (\mathrm{O} / \mathrm{H})>8.1$ the typical values are $q_{\mathrm{PAH}} \sim 4 \%$, in the galaxies with lower metallicity, the average value corresponds to $q_{\mathrm{PAH}} \simeq 1 \%$. In our work [16] from the observations of IC 10 we have suspected the existence of a similar correlation between the PAH mass fraction and the metallicity of gas at the level of individual HII regions within one galaxy, and not only in the comparison of different galaxies. We hypothesized that this correlation may reflect the processes of the PAH molecule formation, but not their subsequent evolution. However, the accuracy of the dependence, observed in IC 10 proved to be insufficient to consider this correlation credible. Hence, it is interesting to test whether in VII Zw 403 there occurs a drop in $q_{\mathrm{PAH}}$ with the decreasing metallicity of HII regions.

Figure 4 shows the flux ratio $F_{8 \mu \mathrm{m}} / F_{24 \mu \mathrm{m}}$ for the individual HII zones as a function of metallicity for the two galaxies studied: IC 10 according to Wiebe et al. [16] and VII Zw 403 from the results of this work.

Note that in [16] we used the metallicity value, obtained with the O3N2-method [23]. For consistency, we tried to estimate the relative oxygen abundance in the HII regions of the IC 10 galaxy with the NS-method [26], used above for VII Zw 403. However, the low intensity of the [NII] $\lambda 6548 \AA$ and [OIII] $\lambda 4959 \AA$ lines in the weak HII regions has increased the measurement errors, although the gen- 

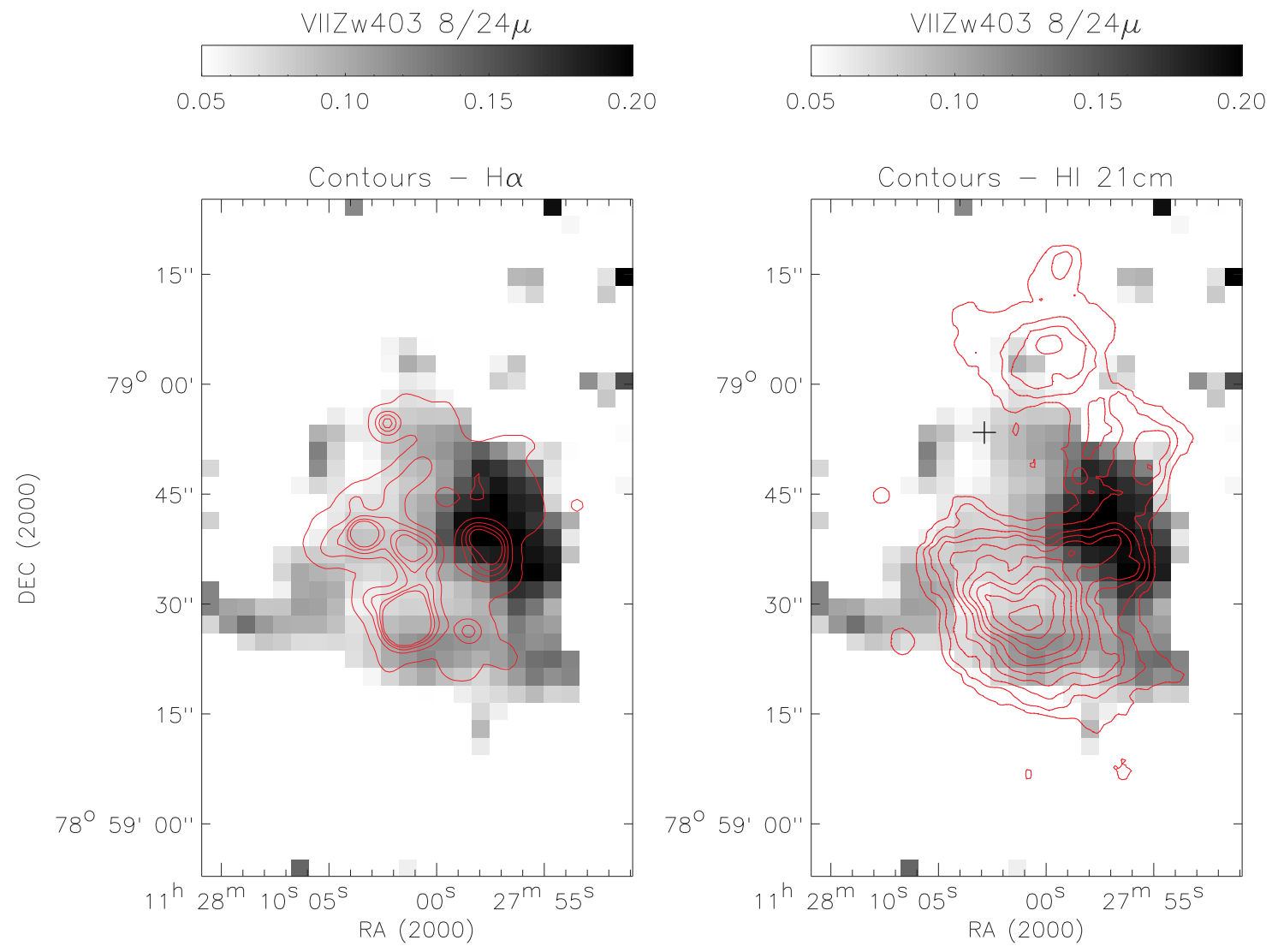

Figure 3. The maps of the flux ratio $F_{8 \mu \mathrm{m}} / F_{24 \mu \mathrm{m}}$ of the VII $\mathrm{Zw} 403$ star-forming region with the superimposed contours in $\mathrm{H}_{\alpha}$ (left) and $\mathrm{HI} 21 \mathrm{~cm}$ (right). The isophotes in the $\mathrm{H}_{\alpha}$ line correspond to the following brightness levels: 500, 1750, 3000, 4250,5500 in arbitrary units; the isophotes in the HI $21 \mathrm{~cm}$ line indicate the brightness levels, corresponding to the beam density of $\mathrm{N}(\mathrm{H})=(5,10,15,20,25,30,35,40,45$ and 50$) \times 10^{20}$ atoms $/ \mathrm{cm}^{2}$. The cross on the right-hand side plot marks the position of a single point X-ray source detected in this galaxy [32].

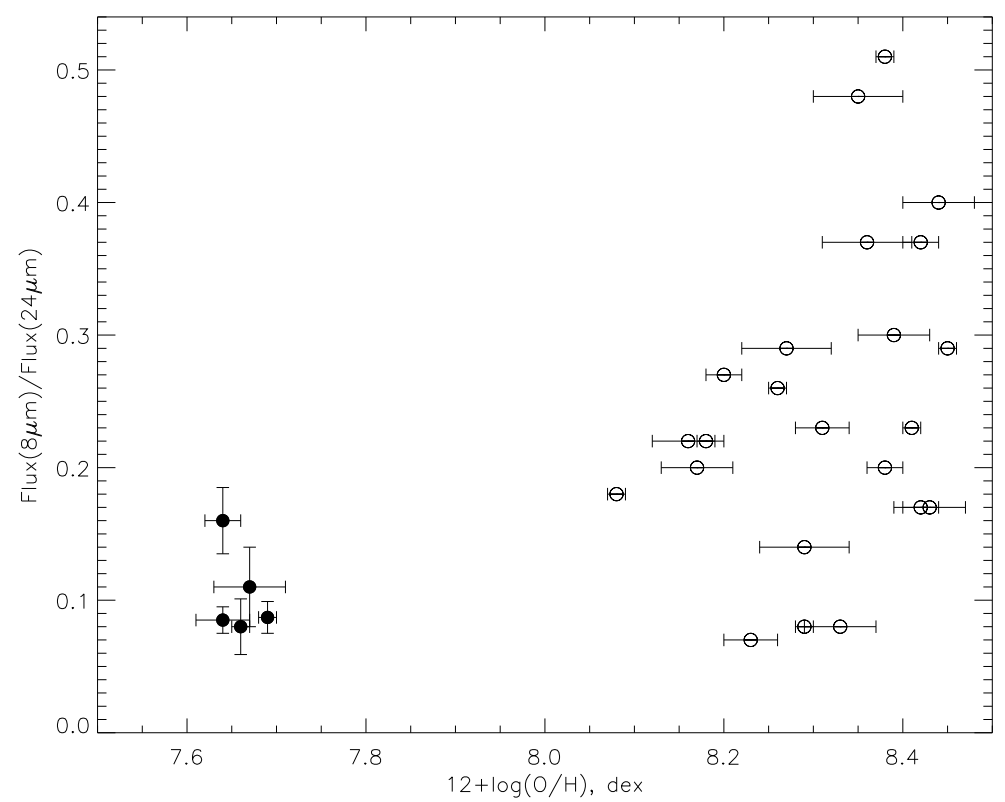

Figure 4. The flux ratio $F_{8 \mu \mathrm{m}} / F_{24 \mu \mathrm{m}}$ depending on the oxygen abundance for individual HII regions based on the results of study of two galaxies-VII Zw 403 (filled circles, according to the present work) and IC 10 (empty circles, according to [16]).

The flux ratio errors for the HII regions in IC 10 do not exceed 0.04 . 
eral form of the dependence has not changed significantly. This is why in Fig. 4 for IC 10 we list the results obtained with the O3N2-method according to the data from [16].

In [16], we noted that the ratio $F_{8 \mu \mathrm{m}} / F_{24 \mu \mathrm{m}}$ apparently drops with decreasing oxygen abundance in the HII regions of IC 10, but the threshold value is not $12+\log (\mathrm{O} / \mathrm{H})=8.0-8.1$, as observed comparing different galaxies, but rather about 8.3. Unfortunately, small variations of metallicity and the ratio $F_{8 \mu \mathrm{m}} / F_{24 \mu \mathrm{m}}$ in the HII regions of VII Zw 403 do not allow to confirm from the observations of this galaxy the presence of the correlation, earlier suspected in IC 10. Such a dependence is only discernible if we consider both galaxies together, but its existence at the level of a comparison between different galaxies has been already known before.

\section{CONCLUSION}

We have analyzed the archival infrared observations of the BCD galaxy VII Zw 403 from the Spitzer Space Telescope and re-analyzed the results of our observations at the 6-m BTA telescope of the SAO RAS with a slit spectrograph and the MPFS, published in [3]. In [3] our estimations of the oxygen, nitrogen and sulfur abundances were based on the assumption that the gas in the bright HII regions is located primarily in the OIII, NII, and SII states. In the present work, we additionally took into account the other stages of ionization, used weaker lines, and made the new metallicity estimates applying several different methods.

Note that the metallicity measurements in individual regions of ionized gas in VII Zw 403 were never previously attempted, except in our earlier work [3].

The oxygen abundance of the individual HII regions and the average value across the galaxy found in the present study and amounting to $12+\log (\mathrm{O} / \mathrm{H})=7.66 \pm 0.03$ is fully consistent with the estimates of the average metallicity in VII $\mathrm{Zw} 403$ determined by other authors: $12+\log (\mathrm{O} / \mathrm{H})=7.73 \pm 0.01$ according to [27]; Nagao et al. [24] from the measurements of [27] have obtained $12+\log (\mathrm{O} / \mathrm{H})=7.7 \pm 0.01$; according to [8] $Z=0.05-0.06 Z_{\odot}$, which corresponds to $12+\log (\mathrm{O} / \mathrm{H})=7.63-7.71$ taken the solar metallicity, adopted in this paper $12+\log (\mathrm{O} / \mathrm{H})_{\odot}=8.93$.

One of the tasks of the iterative analysis of spectroscopic observations, as well as the analysis of infrared images from the Spitzer Space Telescope was the search for the correlation of the PAH mass fraction with the metallicity at the level of individual HII regions that we suspected in the study of the
IC 10 galaxy [16].

However, the variations of metallicity and the ratio $F_{8 \mu \mathrm{m}} / F_{24 \mu \mathrm{m}}$ in the HII regions of VII Zw 403, which can serve as an indicator of the $\mathrm{PAH}$ mass fraction, were small, which did not allow us to confirm the existence of such a correlation within this galaxy.

The analysis of the images in the infrared range has yielded an estimate of the PAH mass fraction averaged across the galaxy amounting to $q_{P A H}<0.5 \%$, what is indicative of the vigorous star formation going on in VII $\mathrm{Zw}$ 403. A comparison of the distribution of $F_{8 \mu \mathrm{m}} / F_{24 \mu \mathrm{m}}$ in the galaxy with an image in the $\mathrm{H}_{\alpha}$ line reveals an elevated PAH abundance at the boundaries and beyond the bright HII regions, and, respectively, a decreased $\mathrm{PAH}$ abundance in their inner regions. A similar effect is also observed in other galaxies, in particular in the Small Magellanic Cloud [15] and IC 10 [16].

A comparison of the map of $F_{8 \mu \mathrm{m}} / F_{24 \mu \mathrm{m}}$ with the emission in the $\mathrm{HI} 21 \mathrm{~cm}$ line has not demonstrated any distinct correlation of the PAH abundance with the distribution of neutral gas in the VII Zw 403 galaxy. Nonetheless, we note that the maximum value of $F_{8 \mu \mathrm{m}} / F_{24 \mu \mathrm{m}}$ is observed towards the western part of the giant dense HI clouds. Perhaps a lower value of $F_{8 \mu \mathrm{m}} / F_{24 \mu \mathrm{m}}$ in the other parts of this cloud is caused by the destruction of PAHs under the influence of ionizing radiation of the $\mathrm{OB}$ associations located there.

The patterns revealed are consistent with the assumption that the PAH molecules form in the giant molecular clouds, to be later destroyed by the ultraviolet radiation.

\section{ACKNOWLEDGMENTS}

The work was conducted with the financial support from the RFBR grant (project № 10-02-00091). O. V. Egorov is grateful for the financial support of the non-profit Dmitry Zimin's Dynasty Foundation and the Federal Target Programm Scientific and Scientific-Pedagogical Cadre of Innovative Russia (state contract 14.740.11.0800). The authors thank the anonymous referee for the helpful comments and V. P. Arkhipova and D. S. Wiebe for the stimulating discussions. The study is based on the observational data obtained at the 6-m SAO RAS telescope, founded by the Russian Ministry of Science (registration № 01-43). We made use of the NASA/IPAC Extragalactic Database (NED), operated by the Jet Propulsion Laboratory on the campus of the California Institute of Technology, under contract with NASA (USA). 
1. Y. Zhao, Q. Gu and Y. Gao, AJ 141, 68 (2011).

2. G. Kauffman, S.D.M. White and B. Guiderdoni, MNRAS 264, 201 (1993).

3. V. P. Arkhipova, T. A. Lozinskaya, A. V. Moiseev and O. V. Egorov, Astron. Rep. 51, 871 (2007).

4. T. A. Lozinskaya, A. V. Moiseev, V. Yu. Avdeev and O. V. Egorov, Astron. Lett. 32, 361 (2006).

5. R. Lynds, E. Tolstoy, E.J. O'Neil and D.A. Hunter, ApJ 116, 146 (1998).

6. R.E. Schulte-Ladbeck, U. Hopp, M.M. Crone and L. Greggio, ApJ 525, 709 (1999).

7. H.H. Loose and T.X. Thuan, in Star-forming Dwarf Galaxies and Related Objects, Ed. by D. Kunth, T.X. Thuan, Tran Thanh Van (Editions Frontieres, Gif-sur-Yvette, 1985), p.73.

8. R.E. Schulte-Ladbeck, U. Hopp, L. Greggio and M.M. Crone, AJ 118, 2705 (1999).

9. T.X. Thuan, J.E. Hibbard and F. Levrier, AJ 128, 717 (2004).

10. T. Ashley and C. Simpson, JSARA (Journal of the Southeastern Association for Research in Astronomy) 2, 30 (2008).

11. C.L. Martin, ApJ 491, 561 (1997).

12. T.X. Thuan, T.B. Williams and E. Malumuth, in Starburst and Galaxy Evolution, Proc. of the 22-th Moriond Astroph. Meeting, Les Arcs, France (Editions Frontieres, Gif-sur-Yvette, 1987), p.151.

13. S. Silich, G. Tenorio-Tagle, C. Munoz-Tunon and L.M. Cairos, AJ 123, 2438 (2002).

14. A.G.G.M. Tielens, Ann. Rev. Astron. Astroph. 46, 289 (2008).

15. K.M. Sandstrom, A.D. Bolatto, B. Draine, et al., ApJ 715, 701 (2010).

16. D. S. Wiebe, O. V. Egorov and T. A. Lozinskaya, Astron. Rep. 55, 585 (2011), arXiv:1102.1060v1 (2011).
17. B.T. Draine, D.A. Dale, G. Bendo, et al., ApJ 663, 866 (2007).

18. V. L. Afanasiev and A. V. Moiseev, Astron. Lett. 31, 194 (2005).

19. V.L. Afanasiev, S.N. Dodonov and A.V. Moiseev, in Stellar dynamics: from classic to modern, Ed. by Osipkov L.P. and Nikiforov I.I. (Saint-Petersburg Univ. press, 2001), p.103.

20. Y.I. Izotov, G. Stasinska, G. Meynet, et al., A\&A 448, 955-970 (2006).

21. M.De Robertis, R. Dufour and R. Hunt, J. Roy. Astron. Soc. Canada 81, 195 (1987).

22. L.S. Pilyugin and T.X. Thuan, ApJ 631, 231-243 (2005).

23. M. Pettini and B.E.J. Pagel, MNRAS 348, 59 (2004).

24. T. Nagao, R. Maiolino and A. Marconi, A\&A 459, 85 (2006).

25. L.S. Pilyugin, J.M. Vílchez and T.X. Thuan, AJ, 720, 1738 (2010).

26. L.S. Pilyugin and L. Mattsson, MNRAS 412, 1145 (2011).

27. Yu.I. Izotov, T.X. Thuan and V.A. Lipovetsky, ApJ Suppl. Ser. 108, 1 (1997).

28. D.R. Garnett, in New views of the Magellanic Clouds, IAU Simposium 190, 266 (1999).

29. M. Peña, G. Stasińska and M.G. Richer, A\&A 476, 745 (2007).

30. C.W. Engelbracht, G.H. Rieke, K.D. Gordon, et al., ApJ 678, 804 (2008).

31. B.T. Draine and A. Li, ApJ 657, 810 (2007).

32. J. Ott, PASP 115, 141 (2003).

Translated by A. Zyazeva 